\title{
Profound Hypotonia and Respiratory Failure due to Suspected Nemaline Myopathy in a Preterm Infant
}

\author{
David A. Bieber, $\mathrm{MD}^{3}$ Jane E. Brumbaugh, $\mathrm{MD}^{2}$ \\ ${ }^{1}$ Department of Pediatric and Adolescent Medicine, Mayo Clinic, \\ Rochester, Minnesota \\ 2 Division of Neonatal Medicine, Department of Pediatric and \\ Adolescent Medicine, Mayo Clinic, Rochester, Minnesota \\ ${ }^{3}$ Division of Child and Adolescent Neurology, Department of \\ Neurology, Mayo Clinic, Rochester, Minnesota
}

Gloria Akuamoah-Boateng, MB, ChB ${ }^{1}$ Raymond C. Stetson, MD ${ }^{2}$ Bethany D. Kaemingk, MD ${ }^{2}$

Address for correspondence Jane E. Brumbaugh, MD, Division of Neonatal Medicine, Mayo Clinic, 200 First Street SW, Rochester, MN 55905 (e-mail: Brumbaugh.Jane@mayo.edu).

AJP Rep 2021;11:e91-e94.

\begin{abstract}
Keywords

- congenital

- floppy

- premature

- hypotonic

- newborn

- actin

Congenital myopathies, such as nemaline myopathy, may present with hypotonia and respiratory failure in the neonatal period. Respiratory function can be further compromised in affected infants by the development of chylous effusions. We present the case of a preterm male infant born at $32^{6 / 7}$ weeks' gestation, who was profoundly hypotonic and required intubation at birth. His clinical course progressed from acute to chronic respiratory failure with mechanical ventilation dependence. He developed bilateral chylous pleural effusions during the newborn period. Whole exome sequencing identified an ACTA1 gene mutation leading to the presumed diagnosis of nemaline myopathy. This case highlights the need to include congenital myopathies in the differential for a preterm newborn with hypotonia and respiratory failure.
\end{abstract}

\section{Case Presentation}

A newborn male was delivered at $32^{6 / 7}$ weeks' gestation following maternal presentation of vaginal bleeding on the day of delivery. The mode of delivery was emergent cesarean section due to fetal bradycardia in the setting of placental abruption. The infant was born to a gravida 2 , para $1-0-0-1$ female in a community hospital with a level I nursery. Parents of the infant were nonconsanguineous and in good health. The pregnancy was complicated by polyhydramnios.

At birth, the infant had no spontaneous movement and an undetectable heart rate. Delivery room resuscitation included positive pressure ventilation, endotracheal intubation, and chest compressions with remote support of a neonatologist via video. The initial arterial blood gas in the first hour of life revealed a mixed respiratory and metabolic acidosis: $\mathrm{pH} 6.92$, partial pressure of carbon dioxide 92, partial pressure of oxygen 92 , base deficit 14 , and bicarbonate 19 . The initial hematocrit was within an acceptable range at $45 \%$. Due to low lung volumes and diffuse hazy opacities consistent with respiratory distress syndrome, surfactant was administered. The critically ill infant then was transported to a level IV neonatal intensive care unit (NICU).

Upon admission, notable physical examination findings included brachycephaly with a broad forehead and temporal narrowing, a prominent nasal tip, bilateral undescended testes, generalized hypotonia, absent suck and rooting reflexes, and depressed palmar and plantar reflexes. He has no contractures or evidence of fractures. Acidosis resolved by 3 hours of life. Laboratory investigations upon admission to the NICU included screening tests for sepsis, inborn errors of metabolism, hypothyroidism; a chromosomal microarray was also sent. Given concern for neurological insult or malformation, head ultrasounds were obtained on days 1 and 4 , which revealed no received

December 1, 2020

accepted after revision

February 23, 2021
DOI https://doi.org/

10.1055/s-0041-1728782. ISSN 2157-6998.

\footnotetext{
(C) 2021. The Author(s).

This is an open access article published by Thieme under the terms of the Creative Commons Attribution-NonDerivative-NonCommercial-License, permitting copying and reproduction so long as the original work is given appropriate credit. Contents may not be used for commercial purposes, or adapted, remixed, transformed or built upon. (https://creativecommons.org/ licenses/by-nc-nd/4.0/) Thieme Medical Publishers, Inc., 333 Seventh Avenue, 18th Floor, New York, NY 10001, USA
} 

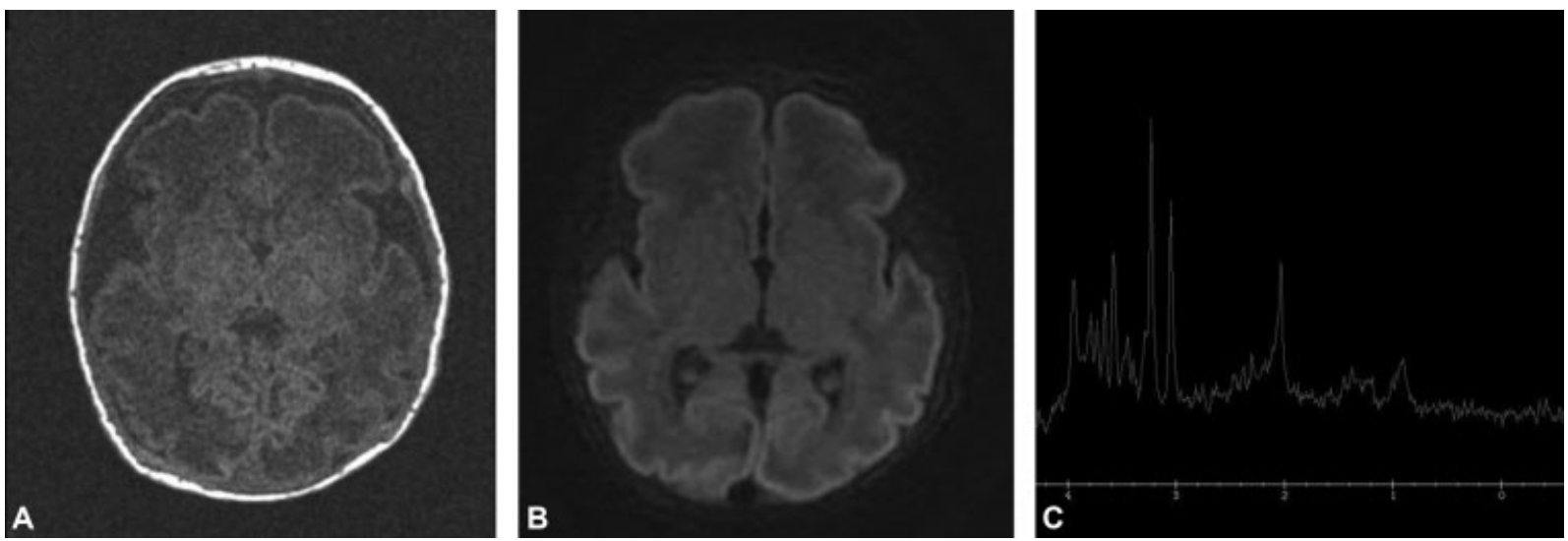

Fig. 1 (A) Magnetic resonance imaging showed no structural abnormalities on T1. (B) Diffusion-weighted imaging did not reveal restricted diffusion. (C) Magnetic resonance spectroscopy had no doublet lactate peak.

intracranial hemorrhage. Due to critically ill status and dysmorphology, an echocardiogram was obtained on day 1 . This demonstrated no structural anomalies, mildly decreased left ventricular systolic function, and moderately decreased right ventricular systolic function. On day 3, noninvasive ventilation was trialed, but the infant required reintubation for invasive mechanical ventilation due to increased work of breathing. He remained hypotonic to a degree that was disproportionate to his postmenstrual age. Magnetic resonance imaging and spectroscopy (MRI/MRS) of the brain on day 9 showed no evidence of hypoxic-ischemic injury, hemorrhage, or structural abnormality (-Fig. 1).

On day 9 , a chest radiograph obtained due to ongoing ventilator dependence demonstrated an incidental, new right-sided pleural effusion ( $\mathbf{- F i g . 2}$ ). Serial chest radiographs showed a worsening right pleural effusion (-Fig. 3), and on day 12 , he underwent an ultrasound-guided diagnostic thoracentesis. The aspirated pleural fluid demonstrated a total nucleated cell count of 9,881 with $97 \%$ lymphocytes and elevated triglycerides at $2,294 \mathrm{mg} / \mathrm{dL}$, findings consistent with a chylothorax. The following week, he developed a left pleural effusion with worsening generalized edema (-Fig. 4). The chylothorax was managed conservatively without thoracotomy tube placement. At 2 weeks of age, neurological examination remained notable for diffuse hypotonia, absent deep tendon reflexes, depressed palmar, and plantar reflex with occasional movements of all extremities. Repeat echocardiogram showed resolution of ventricular dysfunction.

Extensive investigations were performed to elucidate the cause of the infant's persistent hypotonia. The differential diagnosis included sepsis, hypoxic ischemic encephalopathy, myotonic dystrophic, mitochondrial disease, congenital disorder of glycosylation, and congenital myopathy. Thyroid studies were within normal limits. Gene analysis for DMPK revealed no expanded CTG repeats, consistent with a negative result for myotonic dystrophy. There were no reportable alterations identified in the mitochondrial DNA. Due to earlier studies not yielding a diagnosis, whole exome sequencing was sent on a clinical basis and returned on day 27 . Whole exome sequencing revealed a de novo heterozygous variant in the ACTA1 gene $(c .739 G>A)$, a pathogenic mutation in the gene encoding skeletal $\alpha$-actin that is associated with autosomal dominant nemaline myopathy. ${ }^{1}$ Neither parent had symptoms nor signs of myopathy; both parents were tested and neither carried the pathogenic ACTA1 variant.

On day 31, the infant developed signs of cardiovascular compromise with cardiogenic shock requiring vasopressor support. In the setting of deteriorating clinical status, poor prognosis associated with the recently identified underlying myopathy, and discussions with the patient's family, the goals of care were

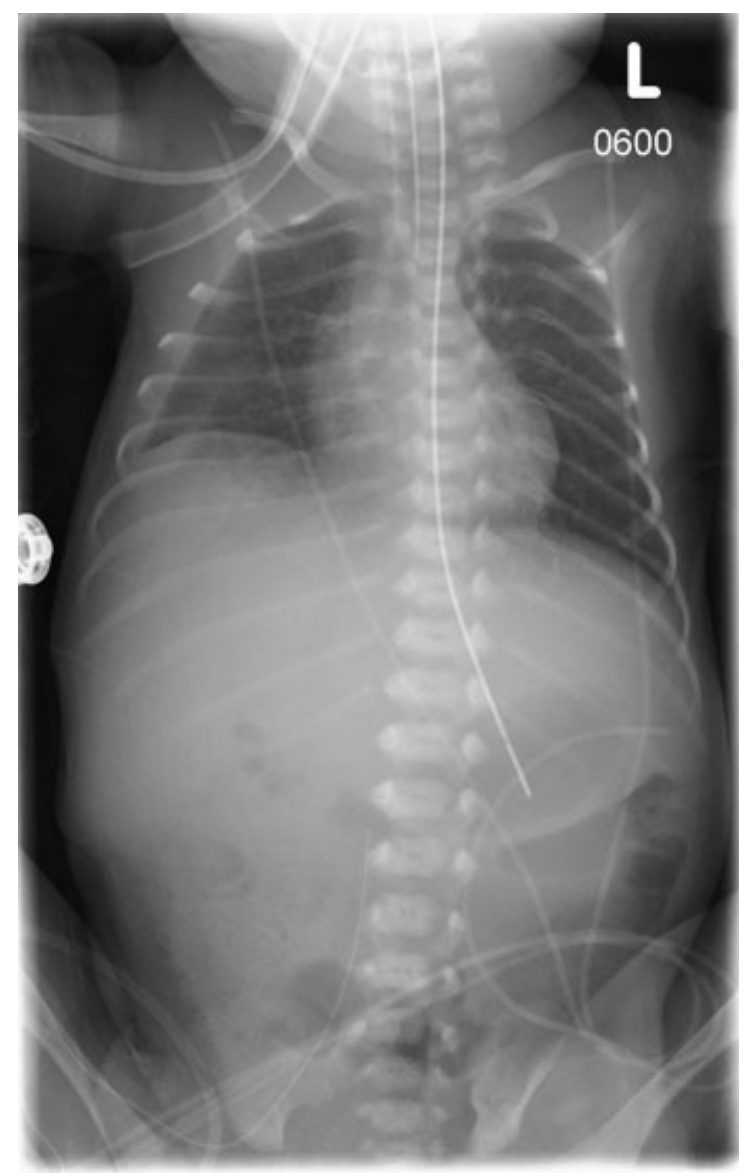

Fig. 2 Right-sided pleural effusion identified was an incidental finding on morning radiograph. 


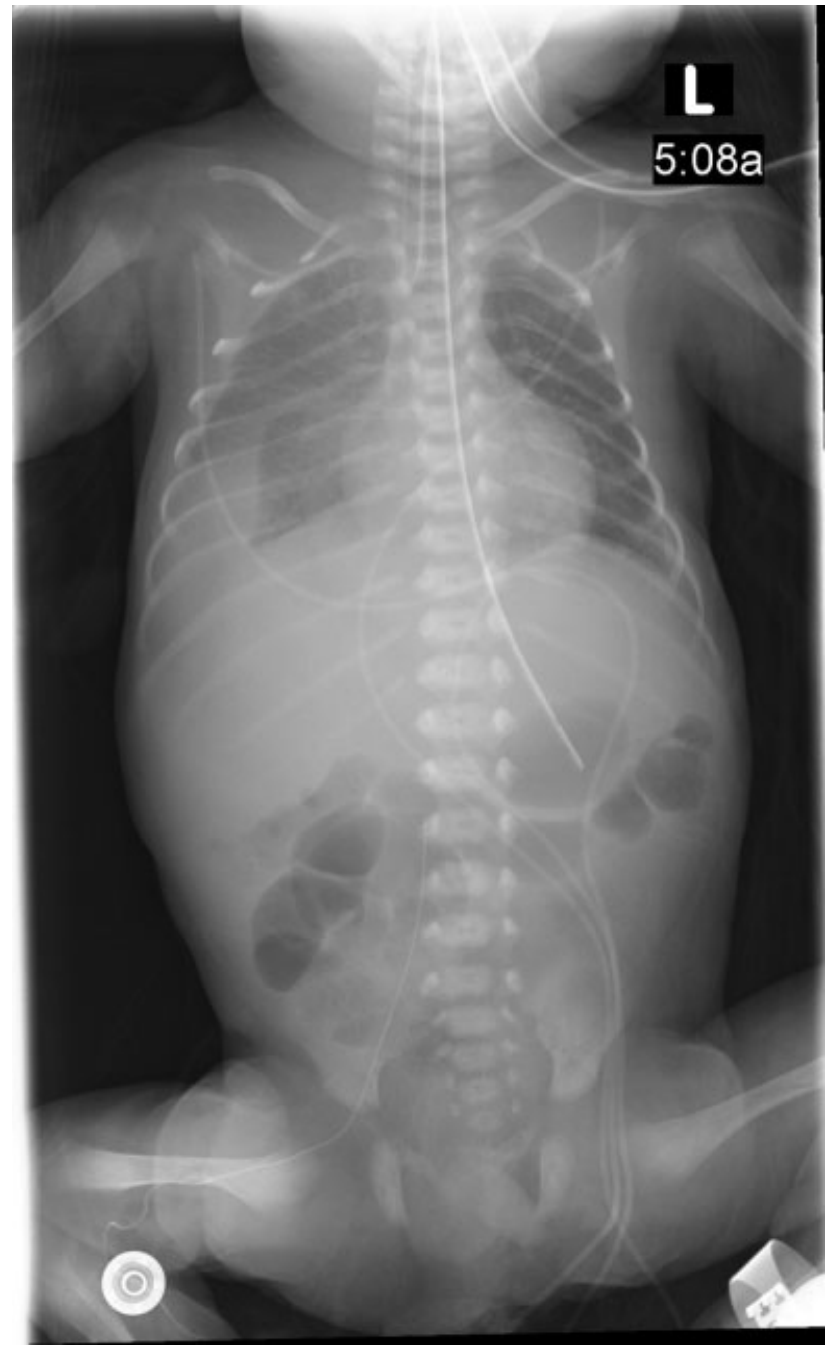

Fig. 3 Progression of right-sided pleural effusion led to diagnostic thoracentesis.

redirected from critical care and life-sustaining measures to intensive comfort measures. The infant was compassionately extubated and died shortly thereafter. Parents declined an autopsy. It is unknown whether cardiac muscle involvement, progressive effusion, or another unidentified morbidity contributed to acute deterioration at the end of life. An autopsy may have been beneficial in investigating the cause of acute deterioration by evaluating for pulmonary lymphangiectasias or other lymphatic malformation and by identifying nemaline bodies on microscopy of muscle tissue to complement the molecular diagnosis of nemaline myopathy. Multiple modalities including muscle MRI, muscle biopsy, and molecular testing may be utilized to arrive at a diagnosis in conjunction with clinical presentation. $^{2-4}$ In the critically ill neonate for whom redirection of goals of care are under consideration, genetic molecular testing may suffice as a noninvasive approach to diagnosis. ${ }^{2}$

\section{Discussion}

The floppy infant is a diagnostic challenge given the myriad of etiologies. Hypotonia is not a diagnosis, but rather a sign which may result from systemic illness, neurological insult,

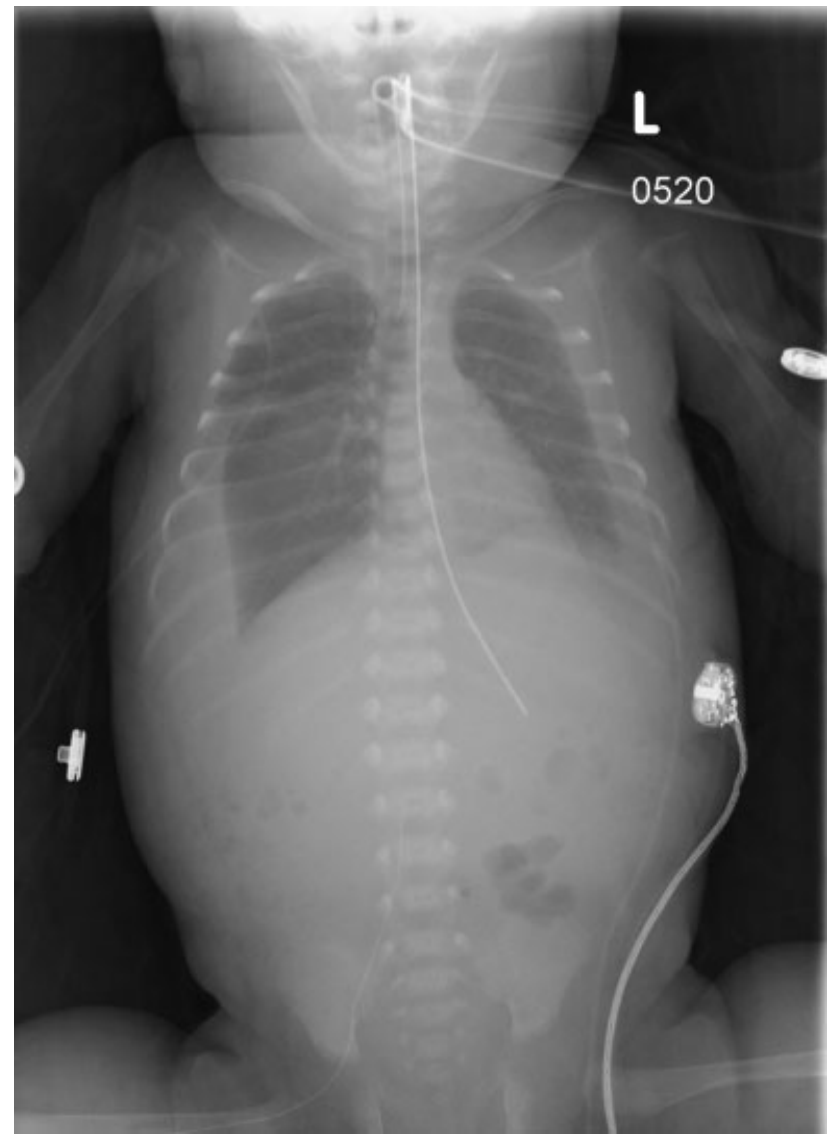

Fig. 4 Bilateral pleural effusions were managed without thoracostomy tube placement.

or congenital abnormalities of the central nervous system, peripheral nervous system, or muscles. Hypoxic ischemic encephalopathy may be among the leading differential diagnoses for a hypotonic neonate with a mixed respiratory and metabolic acidosis who requires mechanical ventilation following an emergent delivery due to placental abruption. Gestational age may further complicate the evaluation of hypotonia as preterm neonates have lower muscle tone than full term neonates. A congenital myopathy should be considered in the differential of neonatal hypotonia after more common etiologies, including hypoxia/ischemia, metabolic derangements, sepsis, and structural brain abnormalities have been excluded. The cause of this infant's depressed presentation with profound hypotonia at birth was likely related to his underlying myopathy. The hypotonia was more severe than what could attributable to his preterm gestation, and MRI/MRS did not reveal evidence of hypoxia or ischemia.

The diagnosis of congenital myopathies is challenging given the significant overlap in clinical presentation. Congenital myopathies result from genetic mutations leading to a defect or deficiency in muscle proteins required for optimal muscle development and contractile function. Congenital myopathies, including nemaline myopathy, are nondystrophic and have a nonprogressive or slowly progressive clinical course. ${ }^{2,5} \mathrm{Nema}-$ line myopathy is heterogeneous from a genetic standpoint and has a wide spectrum of phenotypic presentations. The severe congenital form presents with profound hypotonia and 
respiratory failure in the early neonatal period. ${ }^{6}$ Hypotonic neonates may present with respiratory failure at birth. The ACTA1 gene mutation, identified in this infant, is associated with severe clinical presentations of nemaline myopathy. ${ }^{5,7}$

The etiology of respiratory insufficiency in nemaline myopathy is multifactorial, including respiratory muscle weakness, bulbar dysfunction resulting in poor swallowing and aspiration, abnormal control of breathing, and chylous effusions. Chylothorax has been identified as a cause of respiratory failure in infants with nemaline myopathy previously with autopsy-confirmed pulmonary lymphangiectasias in one case. ${ }^{8-10}$ Chylothorax as a comorbidity predisposes affected neonates to other complications, including fluid and electrolyte derangements, coagulopathy, and immunodeficiency. Later in life progressive scoliosis resulting in restrictive lung disease as well as sleep disordered breathing leading to chronic hypoventilation and atelectasis may contribute. ${ }^{6,11}$ Respiratory failure may culminate in right ventricular failure secondary to pulmonary hypertension. Clinicians should be aware that cardiac involvement in nemaline myopathy, although rare, also may cause cardiac dysfunction. ${ }^{11,12}$

\section{Conclusion}

The differential diagnosis for respiratory failure and hypotonia presenting at birth includes congenital myopathies, such as nemaline myopathy. Respiratory failure secondary to nemaline myopathy is multifactorial and can be complicated by chylothorax. Early molecular testing may expedite diagnosis, prevent invasive testing, and facilitate prognostication for newborns presenting with hypotonia suspected to be secondary to genetic or neurological disorders.
Conflict of Interest

None declared.

\section{References}

1 Sewry CA, Laitila JM, Wallgren-Pettersson C. Nemaline myopathies: a current view. J Muscle Res Cell Motil 2019;40(02):111-126

2 North KN, Wang CH, Clarke N, et al; International Standard of Care Committee for Congenital Myopathies. Approach to the diagnosis of congenital myopathies. Neuromuscul Disord 2014;24(02):97-116

3 Cassandrini D, Trovato R, Rubegni A, et al; Italian Network on Congenital Myopathies. Congenital myopathies: clinical phenotypes and new diagnostic tools. Ital J Pediatr 2017;43(01):101

4 Sanoudou D, Frieden LA, Haslett JN, et al. Molecular classification of nemaline myopathies: "nontyping" specimens exhibit unique patterns of gene expression. Neurobiol Dis 2004;15(03):590-600

5 Malfatti E, Romero NB. Nemaline myopathies: state of the art. Rev Neurol (Paris) 2016;172(10):614-619

6 Ryan MM, Schnell C, Strickland CD, et al. Nemaline myopathy: a clinical study of 143 cases. Ann Neurol 2001;50(03):312-320

7 Agrawal PB, Strickland CD, Midgett C, et al. Heterogeneity of nemaline myopathy cases with skeletal muscle alpha-actin gene mutations. Ann Neurol 2004;56(01):86-96

8 Waisayarat J, Suriyonplengsaeng C, Khongkhatithum C, Rochanawutanon M. Severe congenital nemaline myopathy with primary pulmonary lymphangiectasia: unusual clinical presentation and review of the literature. Diagn Pathol 2015;10:27

9 Schröder JM, Durling H, Laing N. Actin myopathy with nemaline bodies, intranuclear rods, and a heterozygous mutation in ACTA1 (Asp154Asn). Acta Neuropathol 2004;108(03):250-256

10 Garcia-Angarita N, Kirschner J, Heiliger M, et al. Severe nemaline myopathy associated with consecutive mutations E74D and H75Yon a single ACTA1 allele. Neuromuscul Disord 2009;19(07):481-484

11 Wang $\mathrm{CH}$, Dowling JJ, North $\mathrm{K}$, et al. Consensus statement on standard of care for congenital myopathies. J Child Neurol 2012; 27(03):363-382

12 Finsterer J, Stöllberger C. Review of cardiac disease in nemaline myopathy. Pediatr Neurol 2015;53(06):473-477

Funding

None. 